\title{
Expanding the clinical and mutational spectrum of the Ehlers-Danlos syndrome, dermatosparaxis type
}

\author{
Tim Van Damme, MD, Alain Colige, PhD², Delfien Syx, PhD¹, Cecilia Giunta, $\mathrm{PhD}^{3}$, Uschi Lindert, PhD³, \\ Marianne Rohrbach, MD, $\mathrm{PhD}^{3}$, Omid Aryani, $\mathrm{MD}^{4}$, Yasemin Alanay, MD, $\mathrm{PhD}^{5}$, \\ Pelin Özlem Simsek-Kiper, MD, PhD ${ }^{6}$, Hester Y. Kroes, MD, PhD ${ }^{7}$, Koen Devriendt, MD, PhD ${ }^{8}$, \\ Marc Thiry, PhD ${ }^{9}$, Sofie Symoens, $\mathrm{PhD}^{1}$, Anne De Paepe, MD, $\mathrm{PhD}^{1}$ and \\ Fransiska Malfait, MD, PhD ${ }^{1}$
}

\begin{abstract}
Purpose: The Ehlers-Danlos syndrome (EDS), dermatosparaxis type, is a recessively inherited connective tissue disorder caused by deficient activity of ADAMTS-2, an enzyme that cleaves the aminoterminal propeptide domain of types I, II, and III procollagen. Only 10 EDS dermatosparaxis patients have been reported, all presenting a recognizable phenotype with characteristic facial gestalt, extreme skin fragility and laxity, excessive bruising, and sometimes major complications due to visceral and vascular fragility.
\end{abstract}

Methods: We report on five new EDS dermatosparaxis patients and provide a comprehensive overview of the current knowledge of the natural history of this condition.

Results: We identified three novel homozygous loss-of-function mutations (c.2927_2928delCT, p.(Pro976Argfs $\left.{ }^{\star} 42\right)$; c.669_670dupG, p. (Pro224Argfs $\left.{ }^{\star} 24\right)$; and c.2751-2A $>$ T) and one compound heterozygous mutation (c.2T>C, p.? and c.884_887delTGAA, p.(Met295Thrfs26*)) in ADAMTS2 in five patients from four unrelated families. Three of these displayed a phenotype that was strikingly milder than that of previously reported patients.

Conclusion: This study expands the clinical and molecular spectrum of the dermatosparaxis type of EDS to include a milder phenotypic variant and stresses the importance of good clinical criteria. To address this, we propose an updated set of criteria that accurately captures the multisystemic nature of the dermatosparaxis type of EDS.

Genet Med advance online publication 14 January 2016

Key Words: ADAMTS2; ADAMTS-2; dermatosparaxis type; EhlersDanlos syndrome; genotype; phenotype

\section{INTRODUCTION}

Ehlers-Danlos syndrome (EDS) comprises a clinically and genetically heterogeneous group of heritable connective-tissue disorders characterized by skin hyperextensibility and fragility, abnormal wound healing, joint hypermobility, and generalized fragility of the soft connective tissues. Genetic defects affecting the biosynthesis and/or structure of the fibrillar collagens have been implicated in the classic (type V collagen, OMIM 130000), vascular (type III collagen, OMIM 130050), arthrochalasia (type I collagen, OMIM 130060), kyphoscoliosis (lysylhydroxylase 1, OMIM 225400), and dermatosparaxis (ADAMTS-2, OMIM 225410) forms of EDS. Major and minor clinical diagnostic criteria for each of these subtypes were established in 1997 and are known as the Villefranche nosology. ${ }^{1}$

The dermatosparaxis subtype of EDS is an autosomal recessive condition that to date has been described in no more than 10 humans. It was first reported in cattle ${ }^{2,3}$ and subsequently in sheep, ${ }^{4}$ cats, ${ }^{5,6}$ and dogs. ${ }^{7}$ Affected animals display loose and extremely fragile skin (dermatosparaxis means "tearing of the skin"), resulting in large skin lacerations during delivery or early in life, with subsequent infections and premature death. Early ultrastructural studies showed alterations in the dermis of these animals, with loosely packed, thin, and twisted ribbon-like collagen fibrils that displayed a typical "hieroglyphic aspect" on cross-sections, pointing toward impaired collagen biosynthesis and fibrillogenesis. Subsequent biochemical studies performed using dermatosparactic cattle revealed that these abnormal collagen molecules are composed of incompletely processed type I procollagen precursor molecules in which the amino(N)-terminal propeptide $(\mathrm{pN})$ is insufficiently cleaved. ${ }^{2}$ Deficient activity of ADAMTS-2, an endopeptidase that excises the $\mathrm{N}$-propeptide of procollagen chains, was eventually demonstrated. ${ }^{8}$ Although animal dermatosparaxis was the first recognized collagen disorder, it took more than 20 years to confirm the existence of a human counterpart for the disorder. In 1992, three infants were reported with clinical signs resembling

${ }^{1}$ Center for Medical Genetics, Ghent University Hospital, Ghent, Belgium; ${ }^{2}$ Laboratory of Connective Tissues Biology, Tour de Pathologie, GIGA-Cancer, University of Liège, Liège, Belgium; ${ }^{3}$ Division of Metabolism, Connective Tissue Unit, University Children's Hospital and Children's Research Centre, Zurich, Switzerland; ${ }^{4}$ Department of Medical Genetics, Special Medical Center, Tehran, Iran; ${ }^{5}$ Pediatric Genetics Unit, Department of Pediatrics, Acibadem University School of Medicine, Istanbul, Turkey; ${ }^{6}$ Department of Pediatric Genetics, Faculty of Medicine, Hacettepe University, Ankara, Turkey; ${ }^{7}$ Department of Medical Genetics, University Medical Center Utrecht, Utrecht, The Netherlands; ${ }^{8}$ Center for Human Genetics, University Hospitals Leuven, KU Leuven, Leuven, Belgium; ${ }^{9}$ LLaboratory of Cell and Tissue Biology, GIGA-Neurosciences, University of Liège, Liège, Belgium. Correspondence: Fransiska Malfait (Fransiska.Malfait@UGent.be) 
dermatosparaxis. ${ }^{8,9}$ Ultrastructural studies of the dermis demonstrated the same "hieroglyphic" pattern of collagen fibrils as that observed in dermatosparactic animals, and biochemical studies on cultured human fibroblasts confirmed deficient cleavage of the pro $\alpha 1(\mathrm{I})$ and pro $\alpha 2(\mathrm{I}) \mathrm{N}$-propeptides. Seven additional patients were identified subsequently, all of whom displayed a severe phenotype with extremely fragile and lax skin, severe bruising, and characteristic dysmorphic face, which usually lead to a diagnosis within the first few months of life. ${ }^{10-17}$ The clinical findings observed in this small group of patients served as a basis for establishing diagnostic criteria, with severe skin fragility, sagging, and redundant skin as major clinical criteria, and a soft, doughy skin texture, easy bruising, premature rupture of fetal membranes, and large hernias (umbilical, inguinal) as minor criteria. ${ }^{1}$ It took until 1999 to identify the first biallelic mutations in ADAMTS2, the gene encoding ADAMTS-2 (a disintegrin and metalloproteinase with thrombospondin motifs 2), in several patients with the dermatosparaxis type of EDS and in a strain of dermatosparactic calf. ${ }^{17}$

In this paper, we present clinical, biochemical, ultrastructural, and molecular data for five new patients with the dermatosparaxis type of EDS. We expand the clinical phenotype of this disorder by including a much milder clinical and ultrastructural variant that may go undiagnosed in early life, and we review and summarize the current clinical data on human dermatosparaxis to provide the necessary foundation to update the clinical criteria.

\section{Patient cohort}

\section{MATERIALS AND METHODS}

We studied five individuals from four unrelated families who were referred to a medical genetics center because of clinical suspicion of EDS. All patients were investigated by a trained clinical geneticist (F.M., Y.A., M.R., O.A., P.O.S.K., K.D.). Informed consent was obtained from the patients and/or parents participating in this study, and all patients and/or parents consented to the publication of clinical photographs. The study was approved by the Ethics Committee of the Ghent University Hospital, Ghent, Belgium.

\section{DNA and RNA isolation}

Genomic DNA (gDNA) was extracted from blood leukocytes or cultured dermal fibroblasts using the PureGene DNA extraction method according to the manufacturer's instructions (Gentra Systems, Minneapolis, MN). Total RNA was extracted from dermal fibroblasts using the RNeasy method (Qiagen, Venlo, The Netherlands), followed by DNase digestion (RNase-Free DNase set, Qiagen) according to the manufacturer's protocol. For complementary DNA (cDNA) synthesis, the MMLV reverse-transcriptase kit (Invitrogen, Life Technologies, Carlsbad, CA) was used according to the manufacturer's instructions.

\section{Mutation screening}

PCR amplification of ADAMTS2 (NCBI Reference Sequences NG_023212.2 (genomic) and NM_014244.4 (mRNA)) was performed starting from gDNA. Reaction conditions, oligonucleotide primer sequences, and thermal cycling conditions are available on request. PCR products were bidirectionally sequenced using the BigDye Terminator Cycle Sequencing kit protocol (Applied Biosystems, Life Technologies, Carlsbad, CA) and analyzed on an ABI 3730XL Genetic Analyzer (Applied Biosystems). Nucleotide numbering reflects cDNA numbering, with +1 corresponding to the A of the ATG translation initiation codon in the reference sequence. Amino acid residues are numbered from the first methionine residue of the reference sequence (NP_055059.2). The outcome of splice site variants was checked at the cDNA level (primers and conditions available on request). Variant nomenclature follows the HGVS guidelines (http://www.hgvs.org/mutnomen). All variants were checked with Mutalyzer software (https://mutalyzer. $\mathrm{nl} /$ ) and submitted to the EDS Variant Database (https://eds.gene. le.ac.uk/). A unique identifier was obtained for each proband following submission to the EDS Variant Database and is included in the text. Occurrence of the identified variants was assessed using the 1000 Genomes project browser (http://www.1000genomes. org/), the NHLBI Exome Sequencing Project Exome Variant Server database (release ESP6500, http://evs.gs.washington.edu/ EVS/), the Exome Aggregation Consortium (http://exac.broadinstitute.org/), and the EDS Variant database (https://eds.gene.le.ac. uk/home.php?select_db=ADAMTS2).

\section{Expression analysis}

Quantitative PRC (qPCR) primers were designed for ADAMTS2 and three reference genes (HPRT1, RPL13A, and YWHAZ). Total RNA was extracted in triplicate as described, and cDNA was synthesized with the iScript reverse-transcriptase kit (BioRad, Hercules, CA). Assays were performed in triplicate for each sample on a Roche Lightcycler 480 system (Roche, Basel, Switzerland) using Realtime Ready DNA probes Mastermix supplemented with ResoLight Dye (Roche) according to the manufacturer's protocol. Data were analyzed using qbase+ 3.0 (Biogazelle, Zwijnaarde, Belgium).

\section{Biochemical and ultrastructural studies}

A skin biopsy sample was taken from the inner aspect of the upper arm from P2, P3, P4, and P5 for ultrastructural and biochemical studies. Part of the skin biopsy was fixed for $1 \mathrm{hr}$ with $4 \%$ glutaraldehyde in $0.1 \mathrm{~mol} / 1$ Sörensen buffer at $\mathrm{pH} 7.4$, postfixed in $1 \%$ osmium tetroxide, and prepared classically for transmission electron microscopy (TEM). Primary dermal fibroblast cultures were also derived from these skin biopsy samples and grown under standard conditions. For each patient, biochemical analysis of fibrillar (pro)collagen chains was performed at least twice as described previously. ${ }^{18}$ In brief, confluent cells were labeled with ${ }^{14} \mathrm{C}$ proline and (pro)collagen proteins were separated on sodium dodecyl sulfate-polyacrylamide gels. The gels were processed for fluorography, dried, and exposed to an X-ray film.

\section{RESULTS}

\section{Case reports}

The clinical features of all patients are illustrated in Figure 1 and are summarized, together with those of previously reported patients, in Table 1. 


\section{Family 1}

Patient 1 (P1; AN_001994), the first child of nonconsanguineous parents of Caucasian origin, was born after a normal pregnancy and delivery at 38 weeks of gestation. Birth weight was 3,150 g. At birth light blue sclerae, a large tongue, and an umbilical hernia were noted. Closure of the fontanels was delayed. At age two and a half, joint hypermobility became evident, with frequent falling and a delay in gross motor development. Parents noted that she bruised easily and that she frequently had slowly healing orofacial skin lacerations, even after minor trauma. During childhood, she had to be stitched in the face approximately 15 times. This eventually resulted in papyraceous scars on the lips and around the mouth, for which surgical lip correction was performed at age 8 . Because her skin fragility became more and more pronounced, she was referred with a suspicion of a classic or dermatosparaxis type of EDS at the age of 13 years. Clinical examination revealed a girl with a height of $152.5 \mathrm{~cm}$ (10th to 25 th percentile), weight of $45.2 \mathrm{~kg}$ (25th to 50 th percentile), arm span of $158 \mathrm{~cm}$, and occipitofrontal circumference (OFC) of $52 \mathrm{~cm}$ (3rd percentile). She presented mild facial dysmorphism with blue-gray sclerae, mild down-slanting palpebral fissures, epicanthic folds, and some redundancy of the skin on the upper eyelids (Figure 1a,d,e). She presented soft, somewhat doughy, and mildly hyperextensible skin and a visible venous pattern on the thorax. She had facial scars, especially around the mouth, chin, and eyebrows (Figure 1a,d,e), but no atrophic scars were present on her trunk or limbs. Joint hypermobility was generalized (Beighton score 7/9). Dental examination revealed hypermobility of the temporomandibular joints, short dental roots, and a supernumerary molar tooth in the left maxilla. Her hands and feet were short and broad, with increased palmar wrinkling (Figure 1a,b,c). She presented moderate myopia. A cardiac ultrasound and skeletal X-ray survey were normal. At age 13 years and 3 months, bone age using the Greulich and Pyle method was assessed to be between 12 and 13 years. She had a severe thoracic skin laceration after minor trauma that required surgical intervention at approximately age 16 years. Postoperative wound healing was slow, and a large atrophic scar remained (Figure 1f). In her teenage years, she developed rectal prolapse with frequent anal bleeding and experienced frequent episodes of gastrointestinal cramps and bloating. Joint pain became more prominent, but she did not sustain any dislocations or fractures. She was last examined at the age of 19 years. Her main symptoms included progressive, chronic, widespread joint pain, fatigue, and almost complete lack of breast development. Menses, which had started at age 14 years, were normal and regular.

Patient 2 (P2), the younger brother of P1, presented with a clinical history highly comparable to his sister's. He was born at term after a normal pregnancy and delivery. At birth, he presented an umbilical hernia but had no dysmorphic features. Delayed closure of the fontanels was noted. At approximately age 12 months, cystic fibrosis was excluded because of growth restriction. During early childhood, he presented myopia, easy bruising, and fragility of the facial skin, with several facial skin lacerations after minor trauma. He did not present skin fragility on other parts of his body. He sustained a few ankle distortions and one traumatic fracture of the foot, but no dislocations. At the age of 10 years, an osteoid osteoma in the diaphysis of the left tibia was diagnosed. Clinical examination at age 11 years revealed a height of $132 \mathrm{~cm}$ (3rd percentile), weight of $26.5 \mathrm{~kg}$ (below 3rd percentile), arm span of $129 \mathrm{~cm}$, and an OFC of $51 \mathrm{~cm}$ (25th to 50th percentile). His facial appearance was comparable to his sister's (Figure 1g,i), with prominent lips and several papyraceous facial scars (Figure 1g,i). He had milky-white, translucent, and soft skin. Skin hyperextensibility was more pronounced than that of his sister. A small umbilical hernia was noted (Figure 11). He also presented joint hypermobility (Beighton score 6/9), a mild pectus excavatum (Figure 1g), and short and broad hands and feet with increased palmar wrinkling (Figure 1g,h,j,k). Echocardiography was normal, and assessment of bone age showed a delay of 2 to 3 years in skeletal age at the age of 11 years (Greulich and Pyle method). There were no signs of osteopenia. At approximately age 16 years, he developed severe rectal prolapse with profuse bleeding on defecation.

\section{Family 2}

Patient 3 (P3; AN_001995) is the third child of nonconsanguineous healthy parents of Caucasian origin. His two older brothers are healthy. He was born after a normal pregnancy and delivery at 41 weeks of gestation. Birth weight and OFC were $3,150 \mathrm{~g}$ and $32 \mathrm{~cm}$, respectively. Length at the age of 6 weeks was $53.5 \mathrm{~cm}$ (25th percentile). A very large anterior fontanel, a small umbilical hernia, light blue sclerae, and severe palmar wrinkling of the hands were noted at birth. Gross motor development was delayed; he started walking at the age of 23 months. As he became more active, easy bruising, even after minor trauma, and skin and mucosal fragility became apparent. There was marked swelling of the skin on minor trauma, and wound healing was mildly delayed. He had early-onset high myopia (OS $-5 \mathrm{dpt}$; OD $-6 \mathrm{dpt}$ ) that was first noted at the age of 3 years. Cardiac ultrasound and skeletal X-ray examination were normal.

At the age of 3 years, he was referred with a suspicion of the classic or dermatosparaxis type of EDS, but his short stature also evoked a working diagnosis of achondrodysplasia. Clinical examination showed a height of $84.5 \mathrm{~cm}$ (below third percentile), weight of $11.2 \mathrm{~kg}$ (below third percentile), and $\mathrm{OFC}$ of $50 \mathrm{~cm}(\mathrm{P} 50)$. He presented mild facial dysmorphism with a high forehead, flat facial appearance, blue sclerae, mildly down-slanting palpebral fissures, hollow cheeks, low-set ears, telecanthus, and epicanthic folds (Figure $1 \mathrm{~m}-\mathbf{p}$ ). He had micrognathia, full lips, gingival hyperplasia, and small, pointy teeth (Figure 1m-p). His skin was very soft and doughy to the touch, with generalized hyperextensibility and some redundant skin folds at the wrists and the ankles (Figure $\mathbf{1 m - o}$ and q-s). Several ecchymoses and a small umbilical hernia were noted (Figure $\mathbf{1 m - 0}$ ). His hands and feet were very short, with increased palmar wrinkling (Figure $1 \mathbf{m}-\mathbf{o}$ and $\mathbf{q}-\mathbf{s}$ ). 

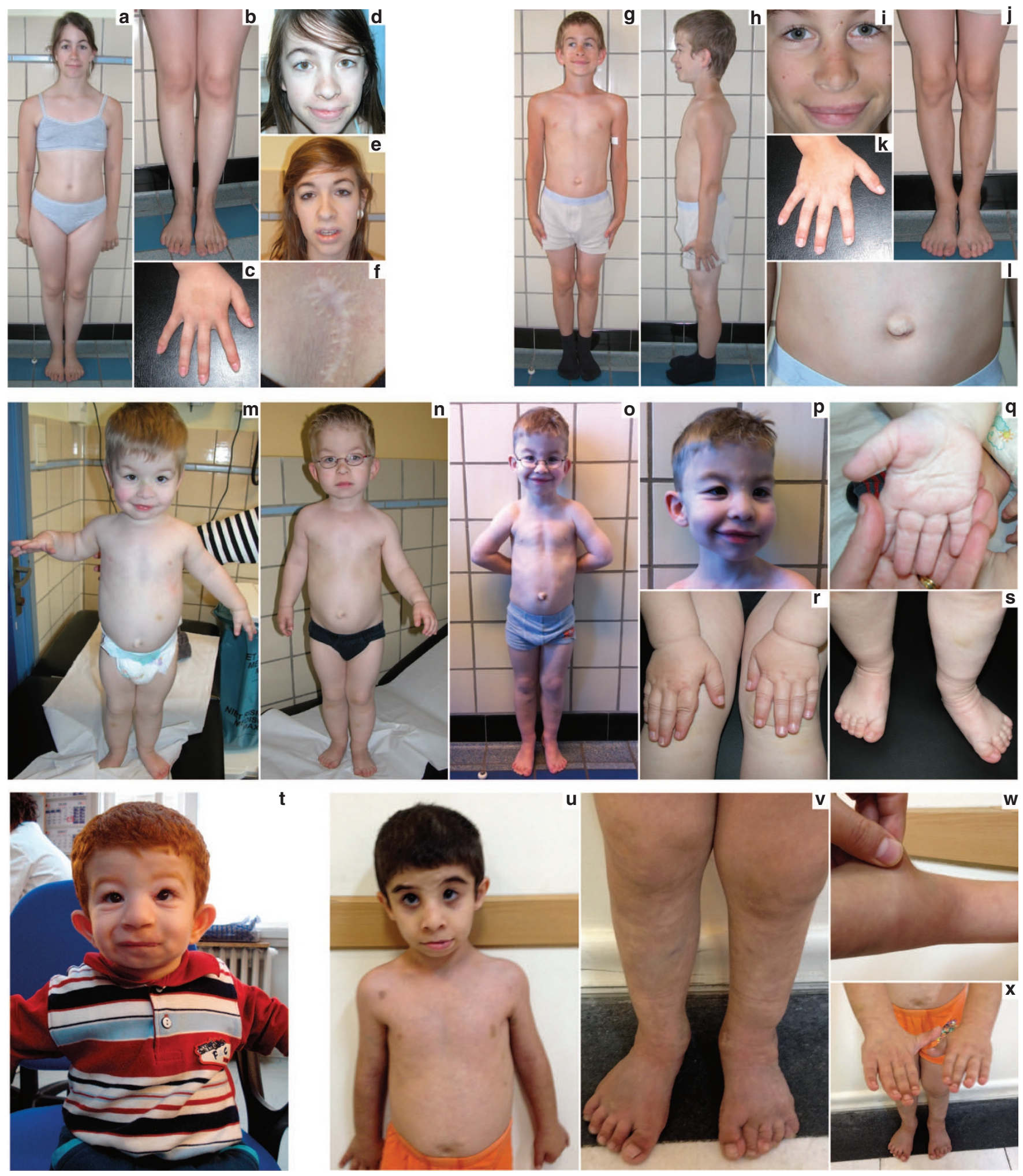

Figure 1 Clinical spectrum of the dermatosparaxis type of the Ehlers-Danlos syndrome. Clinical pictures of P1 at age 13 (a-d) and age 19 (e, f) years, P2 at age 10 (i) and age $11(\mathbf{g}, \mathbf{h}, \mathbf{j}-\mathbf{I})$ years, P3 at age $3(\mathbf{m}, \mathbf{q}), 4(\mathbf{n}, \mathbf{r}-\mathbf{s})$, and $6(\mathbf{o}, \mathbf{p})$ years, P4 at age 2 (t) years, and P5 at age $7(\mathbf{u}-\mathbf{x})$ years.

Generalized joint hypermobility with a Beighton score of $9 / 9$ was present. On follow-up examinations at the ages of 4 and 6 years, he was doing well and there had been no major complications, but fragility of the orobuccal tissues and easy bruising with pronounced swelling of the skin remained a problem. 
Table 1 Summary of the clinical findings for all reported patients, including those in the current report

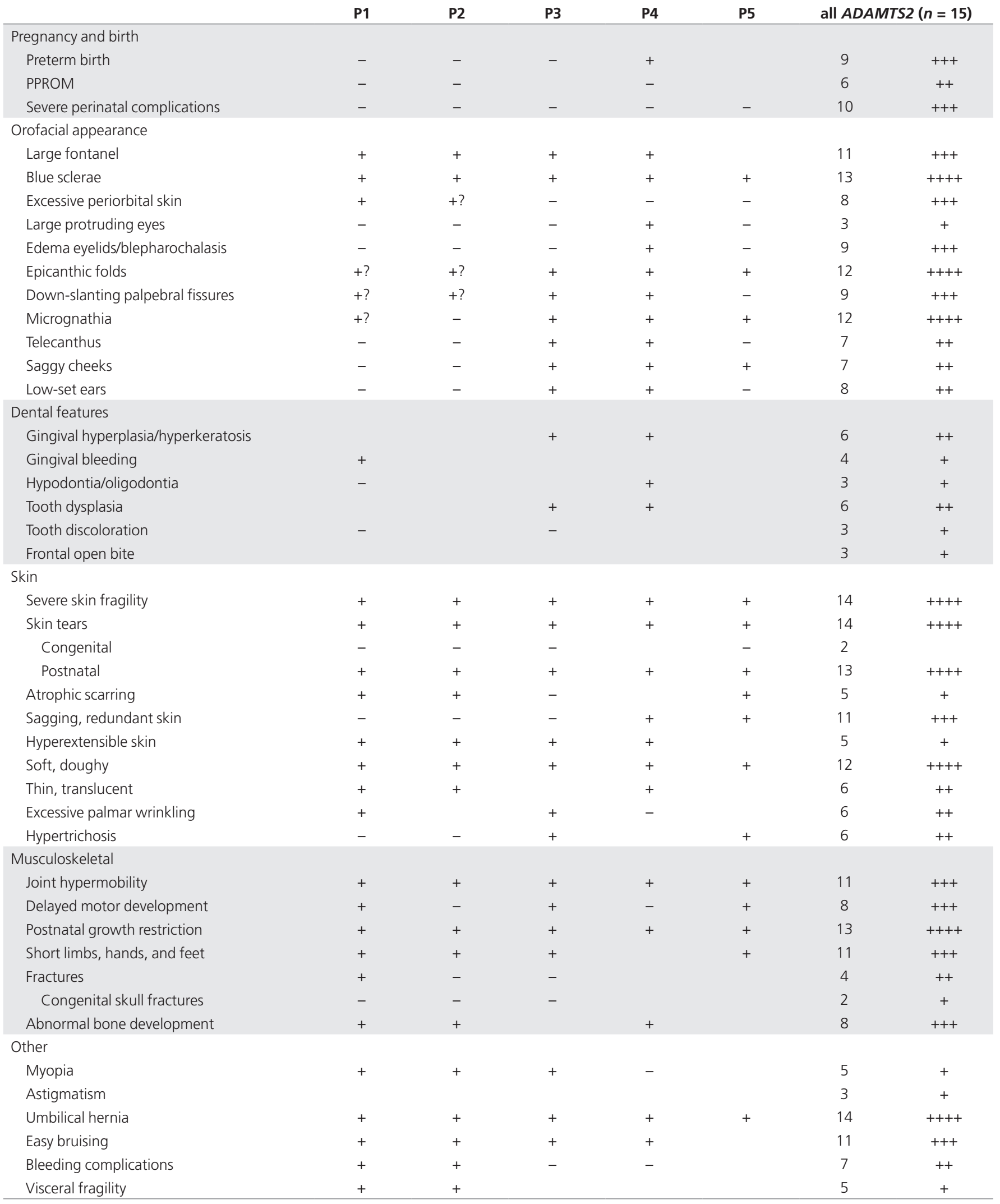

Only features recorded in at least $20 \%$ of patients are included.

Blank, not reported; ++++, present in more than $80 \%$ of patients; +++ , present in more than $60 \%$ of patients; ++ , present in more than $40 \%$ of patients; + , present in more than $20 \%$ of patients or in individual patient reported here; - , absent; + ?, limited presence; PPROM, premature preterm rupture of fetal membranes. 


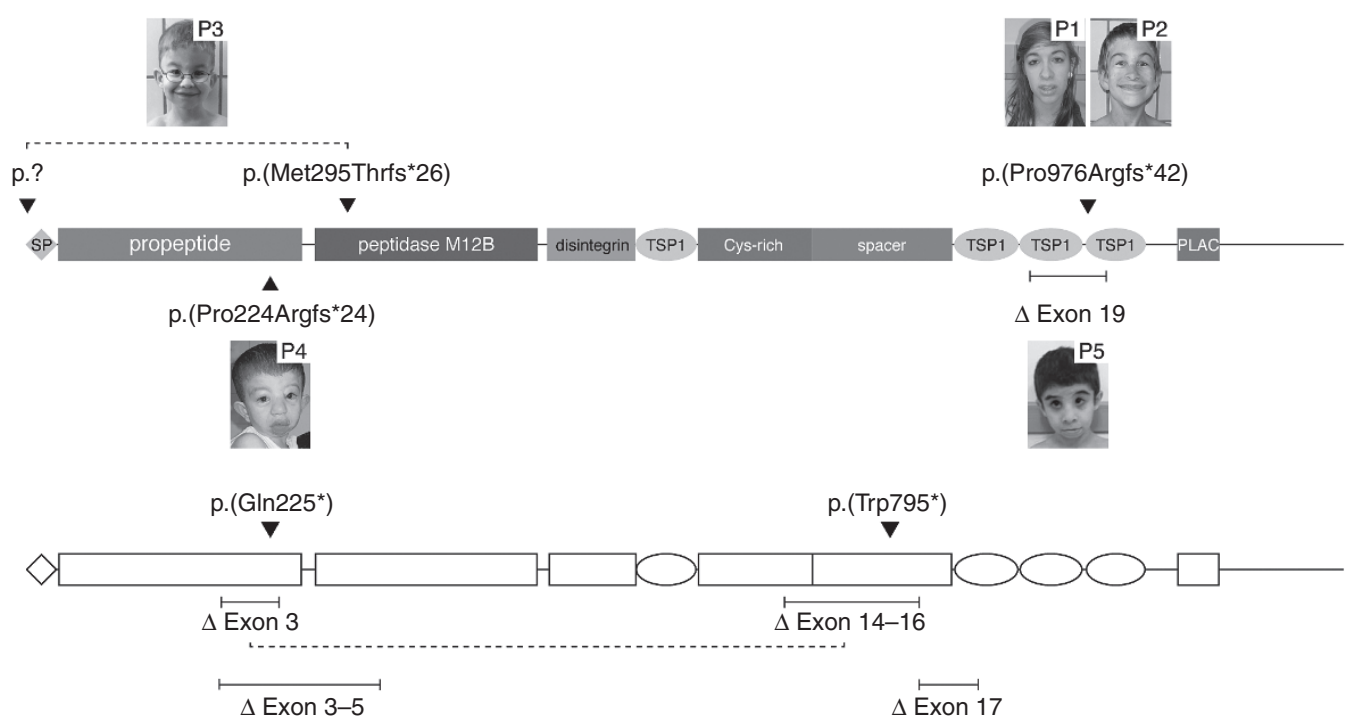

Figure 2 Schematic representation of the domain structure of ADAMTS-2. The mutations reported here are depicted on top. The lower diagram gives an overview of all previously reported mutations. ${ }^{8,13-15,17}$ PLAC, protease and lacunin domain; SP, signal peptide; TSP1, thrombospondin type 1 domain.

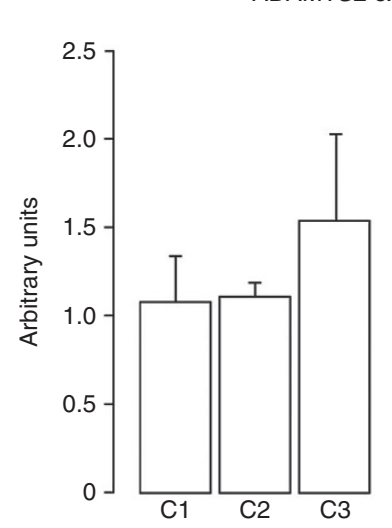

\section{C}

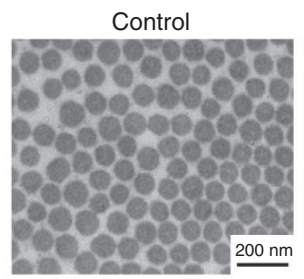

P3

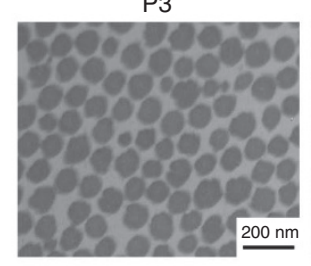

P2

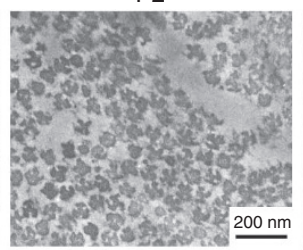

P4

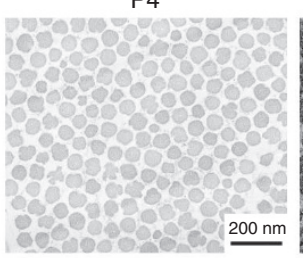

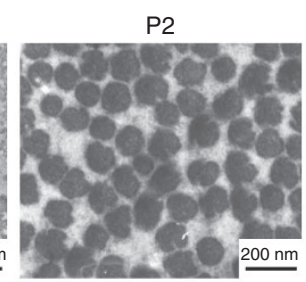

P5

b
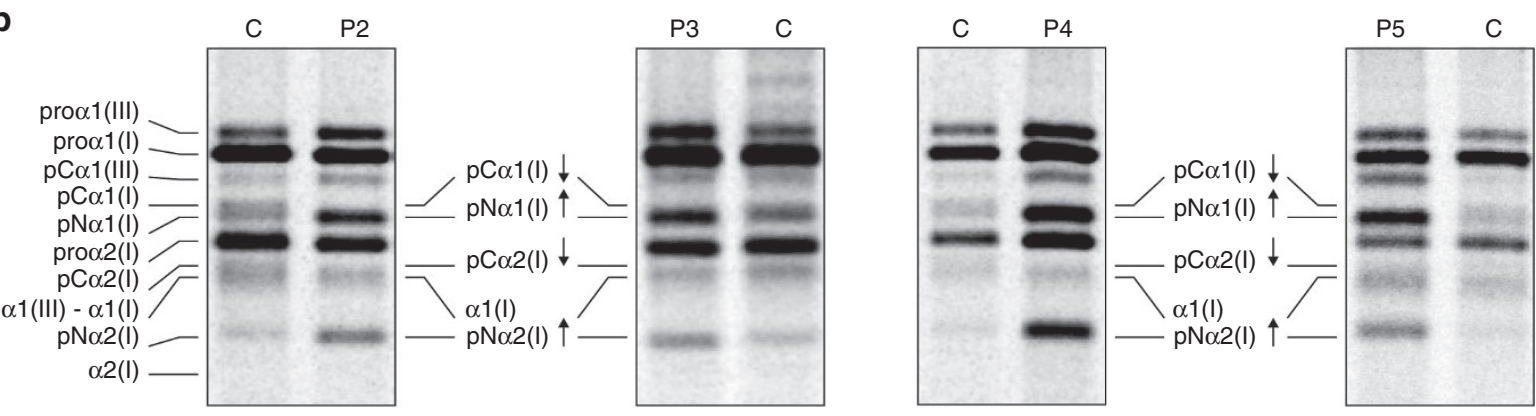

Figure 3 Functional effects of ADAMTS2 mutations. (a) qPCR analysis of P2, P3, P4, and three control individuals shows severely reduced ADAMTS2 expression in total RNA isolated from affected individuals' skin fibroblast cultures. Total RNA was not available for P5 due to growth arrest of the patient-derived fibroblast cell line. Data are expressed as mean \pm SEM. (b) Biochemical electrophoretic analysis of procollagens. Accumulation of pNo1(I) and pNo2(I) procollagen chains and nearly complete absence of bands representing the $\mathrm{pC} \alpha 1(\mathrm{I})$ and $\mathrm{pC} \alpha 2(\mathrm{I})$ procollagen chains were observed in P2, P3, P4, and P5. Arrows denote increased $(\uparrow)$ or decreased $(\downarrow)$ intensity of the respective (pro)collagen fraction. (c) Ultrastructural analysis of dermal skin biopsy samples in P2, P3, P4, and P5 showed relatively mild abnormalities compared to the "hieroglyphic" abnormalities in previously reported patients. qPCR, quantitative polymerase chain reaction.

\section{Family 3}

Patient 4 (P4; AN_001996) is the first child of healthy consanguineous Turkish parents. He was born at 35 5/7 weeks of gestation via cesarean delivery due to breech position. His birth weight was $2,500 \mathrm{~g}$ (50th-75th percentile). Neuromotor development was within normal limits. He was referred to a clinical 
geneticist at the age of 22 months because of delayed tooth eruption and delayed closure of the anterior fontanel. Physical examination revealed an OFC of $49 \mathrm{~cm}$ (90th percentile), body weight of $12 \mathrm{~kg}$ (50th percentile), and body height of $81 \mathrm{~cm}$ (10th to 25th percentile). He presented with a large fontanel $(5 \times 5 \mathrm{~cm})$, down-slanting palpebral fissures, mild telecanthus, palpebral edema, epicanthic folds, blue sclerae, low-set and floppy ears, saggy cheeks, prominent lips, and scars, especially around the mouth and left eyebrow (Figure 1t). He underwent surgery for lacrimal duct stenosis at the age of 20 months. He had micrognathia, periodontal disease, microdontia, and oligodontia (Figure 1t). He presented hyperextensible, thin, and translucent skin that was very smooth and velvety to the touch. Skin redundancy was noted around the hands and feet, and the skin had a somewhat acrogeric aspect. Wound healing was slow, and he bruised easily. He experienced a laceration of the skin medial to the eye and the upper lip after falling on a coffee table. Generalized joint hypermobility (Beighton score of 9/9), bilateral pedes plani, and muscle hypotonia were also noted. Assessment of bone age revealed a bone age of 6 months at the age of 22 months (Greulich and Pyle). By the age of 6 years, he developed severe glaucoma. At 10 years, he needed surgery for rectal prolapse.

\section{Family 4}

This 7-year-old boy (P5; AN_001997) of Iranian descent was born to first-cousin parents. At birth, he presented severe hypotonia with delayed motor milestones. Physical examination at the age of 7 years revealed an acrogeric appearance with micrognathia, epicanthic folds, thin lips, pinched and thin nose, hollow cheeks, and soft and floppy ears (Figure 1u). He presented blue sclerae and had microcornea $(<10 \mathrm{~mm})$ (Figure 1u). He showed generalized joint hypermobility, with a Beighton score of $7 / 9$, and experienced chronic joint pain and fatigue. His skin was thin, translucent, hyperextensible, and smooth (Figure 1u,w). He presented acrogeria of the hands and feet, with some redundant skin folds (Figure 1 $\mathbf{u}, \mathbf{v}, \mathbf{x}$ ). He had extensive bruising and slow wound healing with widened atrophic scars, and he developed varicose veins as early as age 7 years. Mild kyphoscoliosis developed during the first years of life. In addition, he had short hands and feet (Figure 1v, x), a hiatal and umbilical hernia, rectal prolapse, generalized osteopenia, and mitral-valve prolapse.

\section{Molecular identification of ADAMTS2 defects}

Molecular analysis of ADAMTS2 in the siblings $\mathrm{P} 1$ and $\mathrm{P} 2$ identified a homozygous 1-bp deletion in exon 19 (c.2927_2928delCT, p.(Pro976Argfs $\left.\left.{ }^{\star} 42\right)\right)$. This frameshift mutation, located at the beginning of the region encoding the fourth thrombospondin type 1 (TSP1) domain, introduces a premature termination codon (PTC) 41 positions downstream. Their healthy parents were heterozygous for this mutation. P3 was first tested for achondrodysplasia (FGFR3) and the classic type of EDS (COL5A1 and COL5A2). Both analyses were normal, and he was subsequently shown to be compound heterozygous for a start codon mutation (c.2T>C, p.?) and a 4-bp deletion in exon
4 (c.884_887delTGAA, p.(Met295Thrfs $\left.{ }^{\star 26}\right)$ ) in ADAMTS2. The latter shifts the reading frame and introduces a PTC in the region encoding the catalytic domain. His father and mother, respectively, harbored the missense mutation and the 4-bp deletion in a heterozygous state. In P4, molecular analysis identified a homozygous 1-bp duplication in exon 4 (c.669_670dupG) that disrupts the reading frame and produces a truncated transcript (p.(Pro224Argfs $\left.{ }^{\star 24}\right)$ ). DNA from his parents was not available for molecular testing. In P5, a homozygous splice site mutation in intron 18, c.2751-2A>T, was identified. The predicted result, an out-of-frame skipping of exon 19, was confirmed at the cDNA level. None of the mutations were present in the queried databases. The frameshift mutations in P1, P2, P3, P4, and $\mathrm{P} 5$ all produce truncated transcripts that are predicted to be prone to nonsense-mediated mRNA decay (NMD). NMD was confirmed by qPCR analysis that showed severely reduced ADAMTS2 expression (Figure 3a). Figure 2 gives an overview of all ADAMTS2 mutations.

\section{Biochemical analysis}

Sodium dodecyl sulfate-polyacrylamide analysis of pepsindigested fibrillar collagens retained in the cells and secreted in the medium was normal in all affected siblings (data not shown). Procollagen analysis showed the characteristic accumulation of the bands representing $\mathrm{pN \alpha} 1$ (I) and $\mathrm{pN \alpha 2}$ (I) procollagen chains, although the accumulation was variable between patients (Figure 3b). More consistent was the (near) absence of the bands representing $\mathrm{pC} \alpha 1(\mathrm{I})$ and $\mathrm{pC} \alpha 2(\mathrm{I})$ procollagen chains. The bands representing the mature $\alpha 1$ (I) and $\alpha 2$ (I) chains were also consistently absent in all patients, but they were also very faint or absent in control samples. Together, these biochemical findings suggest deficient activity of ADAMTS-2.

\section{Ultrastructural analysis of dermal collagen fibrils}

Collagen fibril architecture was studied using TEM of the reticular dermis in skin biopsy samples from P2, P3, P4, and P5 (Figure 3c). On cross-section, collagen fibers normally show evenly packed and cylindrical collagen fibrils with regular contours. The collagen fibrils in P2 and P5 had very irregular contours and a branched appearance with irregular interfibrillar spaces, but they more or less maintained their cylindrical appearance. This pattern strongly resembled the one described for EDS VIIA. ${ }^{19}$ It should be noted that the level of abnormality varied between different regions within the same biopsy (as illustrated in P2), but in general the abnormalities were less severe than the pathognomonic "hieroglyphic" pattern that was observed in previously reported EDS dermatosparaxis patients. Longitudinal sections (data not shown) showed twisted collagen fibrils in P2, P4, and P5 (data not available for P3).

\section{Overview of the clinical data of all EDS dermatosparaxis patients}

Our report on five additional EDS dermatosparaxis patients brings the total number of molecularly proven human patients 
Table 2 Villefranche diagnostic criteria and updated set of clinical criteria for the dermatosparaxis type of EDS

\begin{tabular}{|c|c|}
\hline Villefranche criteria & Proposed updated criteria \\
\hline Major diagnostic criteria & Severe skin fragility \\
\hline Severe skin fragility & Sagging, redundant skin \\
\hline \multirow[t]{2}{*}{ Sagging, redundant skin } & Bruising easily \\
\hline & $\begin{array}{l}\text { Typical facial gestalt, congenital and/or postnatal, progressive, with swelling of the periorbital soft tissues, blue } \\
\text { sclerae, down-slanting palpebral fissures, epicanthic folds, micrognathia, delayed fontanel closure, and dental } \\
\text { anomalies }\end{array}$ \\
\hline Minor diagnostic criteria & Postnatal growth restriction \\
\hline Bruising easily & Joint hypermobility \\
\hline $\begin{array}{l}\text { Premature rupture of fetal } \\
\text { membranes }\end{array}$ & Umbilical hernia \\
\hline $\begin{array}{l}\text { Large hernias (umbilical, } \\
\text { inguinal) }\end{array}$ & Visceral and vascular fragility, congenital or postnatal \\
\hline
\end{tabular}

PPROM, premature preterm rupture of fetal membranes.

to 15 (14 unrelated families). ${ }^{8,10-17}$ To create the foundation for an update of the clinical criteria, we provide a comprehensive overview of their clinical features (Table 1).

The sex ratio was 11:4 (male:female). Three patients were born to known consanguineous parents. Mean age at diagnosis was 62 months (range: birth to 13 years). Clinical follow-up into puberty and adulthood is reported for only two patients: $\mathrm{P} 2$ in this study and one patient reported by Reardon and colleagues. ${ }^{10}$

Preterm birth was reported for nine patients and was preceded by premature preterm rupture of fetal membranes (PPROM) for six. Mean gestational age was 34 weeks and 4 days $(n=14$, range: 28 to 41 weeks). The umbilical cord was noted to be friable in two infants; one of them also had a short umbilical cord that ruptured after clamping. Perinatal complications were reported in several patients. One patient died shortly after birth (39 weeks of gestation) due to severe hemorrhage and shock. ${ }^{15} \mathrm{~A}$ boy with a gestational age of 33 weeks was born with multiple skull fractures and extensive subgaleal hemorrhage. A dural tear at the site of the skull fracture led to the development of large cerebrospinal fluid collection, and he died due to secondary infection at 145 days of age. ${ }^{14}$ Three other prematurely born infants were admitted to a neonatal intensive care unit for several weeks for a range of complications, including cerebral hemorrhage $(n=2)$, pneumothorax and respiratory distress $(n=1)$, hydronephrosis $(n=1)$, and hypoglycemia, hypocalcemia, and hypothyroidism $(n=1)$.

Most patients, including $\mathrm{P} 4$ and to a lesser extent P3, were born with a severe and recognizable facial gestalt, including prominent and protuberant eyes with puffy, edematous eyelids and excessive periorbital skin, large fontanels and/or wide cranial sutures, a hypoplastic chin, and blue or gray discoloration of the sclerae $(n=12)$. Less frequent findings included gingival hyperplasia $(n=6)$, dental lamina cysts $(n=2)$, and generalized hypertrichosis $(n=6)$. These patients also presented extreme skin fragility with tearing of the skin either at birth $(n=2)$ or within the first few years of life $(n=10)$. In addition, they had lax and sagging skin with redundant skin folds, especially in the neck and around wrists and ankles $(n=10)$. Together, these findings led to an early diagnosis within this group (mean age at diagnosis within this group: 13.8 months; range: birth to 24 months). The phenotype of P1, P2, and to a lesser extent P5 was strikingly milder, and no obvious facial dysmorphic features or skin fragility or redundancy was noted at birth. In early childhood, mild dysmorphic features became gradually more apparent but remained mostly limited to blue sclerae, micrognathia, and epicanthic folds. Skin fragility was initially limited to the face, mouth, and oral mucosae, but it became gradually more pronounced at other body parts later in childhood. In the siblings from family 1 , the skin was hyperextensible, rather than lax, and initially evoked a diagnosis of classic EDS. These observations illustrate clinical variability that has not yet been recognized.

Whereas height, weight, and OFC were usually within normal limits at birth, postnatal growth restriction was reported in all patients, except for those who died soon after birth $(n=13)$. Eleven patients presented with nonrhizomelic shortening of the limbs and short, plump hands and feet with stubby fingers and toes. Joint hypermobility was a consistent finding $(n=11)$ but was often mild at birth. It was associated with a mild delay in gross motor development in approximately half of the patients $(n=8)$. Follow-up data in older patients demonstrate that the joint hypermobility becomes more pronounced later. Four patients had a history of fractures, including (congenital) skull fractures in three. Several other skeletal abnormalities were noted, including delayed ossification of the cranial vault $(n=3)$, Wormian bones $(n=2)$, delayed bone age $(n=2)$, and persistence of woven bone in the ribs of one patient. ${ }^{15}$ Osteopenia was reported in only two patients.

Frequently occurring skin features, apart from the skin fragility $(n=14)$ and laxity/hyperextensibility $(n=15)$, included soft and doughy skin texture $(n=12)$, increased palmar wrinkling $(n=6)$, and atrophic scarring $(n=5)$. One of the most consistent clinical findings was an umbilical hernia at birth $(n=14)$. Easy bruising was frequent $(n=11)$ and often very severe, with the formation of large subcutaneous hematomas. Bleeding problems were reported for seven patients and ranged from severe epistaxis and gum bleeding to internal and (congenital) cerebral hemorrhages. Arterial rupture and aortic dilatation have not been reported so far. A 9-year-old girl ruptured her 
diaphragm due to postoperative vomiting. She subsequently developed a paraesophageal hernia with incarceration of the stomach that was further complicated by the occurrence of a large abdominal hematoma after reduction. ${ }^{9,13}$ Two patients had bladder diverticula complicated by spontaneous bladder rupture, and two other patients (P1 and P2 in this study) developed rectal prolapse with profuse anal bleeding during puberty.

The ophthalmic phenotype in dermatosparaxis is relatively mild. One patient had severe congenital myopia, ${ }^{8,16}$ and several others presented with early-onset and progressive myopia $(n=5)$. Three others had astigmatism, and one patient developed severe glaucoma at a very young age (P4 in this study).

In addition to gingival hyperplasia and dental lamina cysts, several other dental abnormalities have been reported. These include microdontia $(n=6)$, agenesis of several permanent teeth $(n=3)$, and tooth discoloration $(n=3)$. Abnormal morphology of the molars $(n=2)$ and severe enamel attrition of the deciduous teeth $(n=2)$ have been reported in a limited number of patients. ${ }^{13,20}$

\section{DISCUSSION}

The dermatosparaxis type of EDS is a rare but severe autosomal recessive heritable connective-tissue disorder resulting from disturbed processing of type I procollagen $\mathrm{N}$-propeptide due to biallelic loss-of-function mutations in ADAMTS2 encoding the most important type I procollagen $\mathrm{N}$-endopeptidase. To date only 10 unrelated patients with ADAMTS2 mutations have been identified, and we now report five additional patients from four unrelated families. In general, the most distinct clinical features (summarized in Table 1) include: (i) a recognizable orofacial gestalt at birth with swelling of the periorbital soft tissues, blue sclerae, down-slanting palpebral fissures, epicanthic folds, micrognathia, delayed fontanel closure, and dental anomalies in childhood and adulthood; (ii) extreme skin fragility and redundant, almost lax, skin with excessive skin folds at the wrists and ankles; (iii) severe susceptibility to bruising with a risk of subcutaneous hematomas and hemorrhage; (iv) postnatal growth restriction; (v) umbilical hernia; (vi) PPROM; (vii) perinatal complications, most notably congenital skull fractures; and (viii) visceral complications due to connective tissue fragility (e.g., bladder rupture, diaphragmic eventration).

This clinical spectrum often evokes a wide differential diagnosis that includes the classic type of EDS (skin fragility), osteogenesis imperfecta (blue sclerae, short stature, skull fractures), cutis laxa (redundant skin with excessive skin folds), and achondrodysplasia (short stature). Whereas most patients, including P3 and P4 in the current study, had a very similar and recognizable phenotype at birth, the siblings from family 1 , and to a lesser extent P5, lacked most of the typical facial features at birth and in infancy, and they did not initially display pronounced skin fragility. Their phenotype did become more pronounced during childhood and eventually aligned with that of previous reports. This demonstrates that milder forms of human dermatosparaxis exist and that this condition can go undiagnosed in a nonspecialized clinical setting. However, long-term follow-up of family 1 also showed the progressive nature of this condition and the need for long-term surveillance. Our observations also stress the need for new and accurate diagnostic criteria. The current criteria, established in 1997 (Table 2), were based on initial observations in a few patients, but these are no longer up-to-date with current knowledge. In Table 2, we propose an updated set of clinical criteria that accurately capture the multisystemic nature of the dermatosparaxis type of EDS by including additional characteristics, such as the distinct orofacial dysmorphology, postnatal growth restriction, congenital skull fractures, and visceral fragility resulting from generalized connective tissue weakness.

The "hieroglyphic" appearance of collagen fibrils on TEM has historically been considered pathognomonic for the dermatosparaxis type of EDS. The relatively mild ultrastructural abnormalities of the collagen fibrils of the dermis reported here, in both mildly (P2, P5) and more severely affected (P3-P4) patients, warn against relying too heavily on TEM. These observations also emphasize the necessity of molecular analysis of ADAMTS2 in the diagnostic work-up of the dermatosparaxis type of EDS.

The first mutations in ADAMTS2 in human and bovine dermatosparaxis were identified more than 15 years ago. ${ }^{17}$ Until now, only five biallelic mutations had been reported in 10 unrelated families, including a recurrent homozygous nonsense mutation p. $\left(\mathrm{Gln} 225^{\star}\right)$ in six patients (refs. 8,14,17), a unique homozygous nonsense mutation p.(Trp795*) (ref. 13), a homozygous in-frame skip of exons three to five (ref. 13), a homozygous in-frame skip of exon 17 (ref. 15), and, finally, compound heterozygosity for an out-of-frame exon-skip of exon three and an in-frame skipping of exons 14 to 16 (ref. 13). Our study describes three novel homozygous loss-of-function mutations (c.2927_2928delCT, p.(Pro976Argfs $\left.{ }^{\star} 42\right)$; c.669670dupG, p.(Pro224Argfs $\left.{ }^{\star} 41\right)$; and c.2751-2A $>$ T) and one compound heterozygous mutation (c.2T>C, p.? and c.888891delTGAA, p.(Met295Thrfs25*)). The milder phenotype in $\mathrm{P} 1, \mathrm{P} 2$, and P5 might be due to the specific genotype in these patients. c.2927_2928delCT (P1-2) and c.2751-2A>T (P5) are both nonsense alleles prone to NMD-mediated degradation, but several studies have shown that some transcripts can escape NMD, rendering it less than $100 \%$ sufficient. ${ }^{21-23}$ As such, these alleles could produce truncated but (partially) active ADAMTS-2 enzymes lacking either the last TSP1 domain and the PLAC (protease and lacunin) domain (P1 and P2) or the two most C-terminal TSP1 domains and the PLAC domain (P5). Investigation of the $\mathrm{N}$-endopeptidase activity of various forms of recombinant ADAMTS-2 has previously shown that removal of these domains results in an enzyme that is still significantly active. ${ }^{24}$ In addition, two other members of the ADAMTS family have been shown to possess type I procollagen $\mathrm{N}$-endopeptidase activity and could (partially) compensate for the reduced activity of ADAMTS-2.25,26 ADAMTS-3 has been proposed as the major type II procollagen-processing enzyme, but it is also highly expressed in many Colla1-expressing tissues during mouse embryonic development and might be 
responsible for some of the type I procollagen $\mathrm{N}$-endopeptidase activity, ${ }^{26,27}$ and ADAMTS-14 has been shown to have type I procollagen I $\mathrm{N}$-endopeptidase activity in vitro. ${ }^{25}$

In line with the role of ADAMTS-2 as an important procollagen type I N-endopeptidase, ${ }^{9,12,17}$ we found abnormal processing of the N-propeptide of type I procollagen, with accumulation of $\mathrm{pN} \alpha 1(\mathrm{I})$ and $\mathrm{pN} \alpha 2$ (I) procollagen chains and near-absence of $\mathrm{pC} \alpha 1(\mathrm{I})$ and $\mathrm{pC} \alpha 2(\mathrm{I})$ procollagen chains in patients' derived fibroblasts. Apart from its activity in type I procollagen, several studies have demonstrated that ADAMTS-2 also possesses $\mathrm{N}$-endopeptidase activity for types II, III, and V procollagen. ${ }^{24,28}$ Sodium dodecyl sulfate-polyacrylamide analysis could not detect accumulation of $\mathrm{pN}$ chains of type III and type $\mathrm{V}$ collagen, but this technique lacks the necessary sensitivity to detect mild changes in collagen processing. Therefore, we cannot exclude that deficient activity towards these substrates adds to the severity of the disorder, such as the extreme skin fragility and laxity (type V collagen) and the propensity for bruising and bleeding (type III collagen).

In conclusion, we have expanded the clinical phenotype of this disorder by describing a much milder phenotypic and ultrastructural variant of the dermatosparaxis type of EDS, highlighted the necessity of molecular diagnosis and the utility of biochemical collagen analysis, and laid the foundation for an update of the clinical criteria.

\section{ACKNOWLEDGMENTS}

D.S. and F.M. are fellows of the Fund for Scientific Research, Belgium. This work was supported by Methusalem Grant 08/01M01108 from Ghent University to Anne De Paepe. Some of the data included in this study were previously presented at the European Human Genetics Conference 2013 (8-11 June 2013, Paris, France) and the 15th Annual Meeting of the Belgian Society of Human Genetics (6 March 2015, Liège, Belgium).

\section{DISCLOSURE}

The authors declare no conflict of interest.

\section{REFERENCES}

1. Beighton P, De Paepe A, Steinmann B, Tsipouras P, Wenstrup RJ. Ehlers-Danlos syndromes: revised nosology, Villefranche, 1997. Ehlers-Danlos National Foundation (USA) and Ehlers-Danlos Support Group (UK). Am J Med Genet 1998;77:31-37.

2. Lenaers A, Ansay M, Nusgens BV, Lapière CM. Collagen made of extended -chains, procollagen, in genetically-defective dermatosparaxic calves. Eur $J$ Biochem 1971;23:533-543.

3. Hanset R, Lapiere CM. Inheritance of dermatosparaxis in the calf. A genetic defect of connective tissues. J Hered 1974;65:356-358

4. Fjolstad M, Helle O. A hereditary dysplasia of collagen tissues in sheep. J Pathol 1974;112:183-188.

5. Holbrook KA, Byers PH, Counts DF, Hegreberg GA. Dermatosparaxis in a Himalayan cat: II. Ultrastructural studies of dermal collagen. J Invest Dermatol 1980;74:100-104.

6. Counts DF, Byers PH, Holbrook KA, Hegreberg GA. Dermatosparaxis in a Himalayan cat: I. Biochemical studies of dermal collagen. J Invest Dermatol 1980;74:96-99.

7. Holbrook KA, Byers PH. Structural abnormalities in the dermal collagen and elastic matrix from the skin of patients with inherited connective tissue disorders. J Invest Dermatol 1982;79 Suppl 1:7s-16s.
8. Smith LT, Wertelecki W, Milstone LM, et al. Human dermatosparaxis: a form of Ehlers-Danlos syndrome that results from failure to remove the aminoterminal propeptide of type I procollagen. Am J Hum Genet 1992;51: 235-244.

9. Nusgens BV, Verellen-Dumoulin C, Hermanns-Lê T, et al. Evidence for a relationship between Ehlers-Danlos type VII C in humans and bovine dermatosparaxis. Nat Genet 1992;1:214-217.

10. Reardon W, Winter RM, Smith LT, Lake BD, Rossiter M, Baraitser M. The natural history of human dermatosparaxis (Ehlers-Danlos syndrome type VIIC). Clin Dysmorphol 1995;4:1-11.

11. Fujimoto A, Wilcox WR, Cohn DH. Clinical, morphological, and biochemical phenotype of a new case of Ehlers-Danlos syndrome type VIIC. Am J Med Genet 1997;68:25-28.

12. Colige A, Nuytinck L, Hausser I, et al. Novel types of mutation responsible for the dermatosparactic type of Ehlers-Danlos syndrome (Type VIIC) and common polymorphisms in the ADAMTS2 gene. J Invest Dermatol 2004;123: 656-663.

13. Malfait F, De Coster P, Hausser I, et al. The natural history, including orofacial features of three patients with Ehlers-Danlos syndrome, dermatosparaxis type (EDS type VIIC). Am J Med Genet A 2004;131:18-28.

14. Bar-Yosef O, Polak-Charcon S, Hoffman C, Feldman ZP, Frydman M, Kuint J. Multiple congenital skull fractures as a presentation of Ehlers-Danlos syndrome type VIIC. Am J Med Genet A 2008;146A:3054-3057.

15. Solomons J, Coucke P, Symoens S, et al. Dermatosparaxis (Ehlers-Danlos type $\mathrm{VIIC})$ : prenatal diagnosis following a previous pregnancy with unexpected skull fractures at delivery. Am J Med Genet A 2013;161A:1122-1125.

16. Wertelecki W, Smith LT, Byers P. Initial observations of human dermatosparaxis: Ehlers-Danlos syndrome type VIIC. J Pediatr 1992;121:558-564.

17. Colige A, Sieron AL, Li SW, et al. Human Ehlers-Danlos syndrome type VII C and bovine dermatosparaxis are caused by mutations in the procollagen I N-proteinase gene. Am J Hum Genet 1999;65:308-317.

18. Nuytinck L, Narcisi P, Nicholls A, Renard JP, Pope FM, De Paepe A. Detection and characterisation of an overmodified type III collagen by analysis of noncutaneous connective tissues in a patient with Ehlers-Danlos syndrome IV. J Med Genet 1992;29:375-380

19. Giunta C, Chambaz C, Pedemonte M, Scapolan S, Steinmann B. The arthrochalasia type of Ehlers-Danlos syndrome (EDS VIIA and VIIB): the diagnostic value of collagen fibril ultrastructure. Am J Med Genet A 2008;146A:1341-1346.

20. De Coster PJ, Malfait F, Martens LC, De Paepe A. Unusual oral findings in dermatosparaxis (Ehlers-Danlos syndrome type VIIC). J Oral Pathol Med 2003;32:568-570.

21. Romão L, Inácio $A$, Santos $S$, et al. Nonsense mutations in the human betaglobin gene lead to unexpected levels of cytoplasmic mRNA accumulation. Blood 2000;96:2895-2901.

22. Asselta R, Duga S, Spena $S$, et al. Congenital afibrinogenemia: mutations leading to premature termination codons in fibrinogen $\mathrm{A}$ alpha-chain gene are not associated with the decay of the mutant mRNAs. Blood 2001;98: 3685-3692.

23. Danckwardt S, Neu-Yilik G, Thermann R, Frede U, Hentze MW, Kulozik AE. Abnormally spliced beta-globin mRNAs: a single point mutation generates transcripts sensitive and insensitive to nonsense-mediated mRNA decay. Blood 2002;99:1811-1816.

24. Colige A, Ruggiero F, Vandenberghe I, et al. Domains and maturation processes that regulate the activity of ADAMTS-2, a metalloproteinase cleaving the aminopropeptide of fibrillar procollagens types I-III and V. J Biol Chem 2005;280:34397-34408.

25. Colige $A$, Vandenberghe I, Thiry $M$, et al. Cloning and characterization of ADAMTS-14, a novel ADAMTS displaying high homology with ADAMTS-2 and ADAMTS-3. J Biol Chem 2002;277:5756-5766.

26. Le Goff C, Somerville RP, Kesteloot F, et al. Regulation of procollagen aminopropeptide processing during mouse embryogenesis by specialization of homologous ADAMTS proteases: insights on collagen biosynthesis and dermatosparaxis. Development 2006;133:1587-1596.

27. Fernandes RJ, Hirohata S, Engle JM, et al. Procollagen II amino propeptide processing by ADAMTS-3. Insights on dermatosparaxis. I Biol Chem 2001:276:31502-31509

28. Wang WM, Lee $S$, Steiglitz BM, et al. Transforming growth factor-beta induces secretion of activated ADAMTS-2. A procollagen III N-proteinase. J Biol Chem 2003;278:19549-19557. 